\title{
Le chichewa: essai de description dans une perspective didactique
}

\section{Allan Lipenga}

\section{Introduction}

Le chichewa est une langue principale du Malawi, en Afrique australe. Selon le recensement de 1998, 57\% de la population Malawienne (soit 5,7 millions d'habitants) utilisent le chichewa comme langue de communication dans la famille. En outre, d'après J. Bresnan et S.A. Mchombo (1987), l'aire chichewaphone couvrirait aussi les pays comme le Mozambique, la Zambie et le Zimbabwe (voir la carte plus bas). Dans ces pays, la langue s'appelle le chinyanja. Ainsi, l'objectif de cette discussion est de tenter de caractériser cette langue dans une visée didactique car l'enseignement du français aux apprenants chichewaphones doit prendre en considération les différences structurales qui séparent les deux langues (H.G.Widdowson, 1981; E. Roulet, 1995).

\section{L'aire chichewaphone}

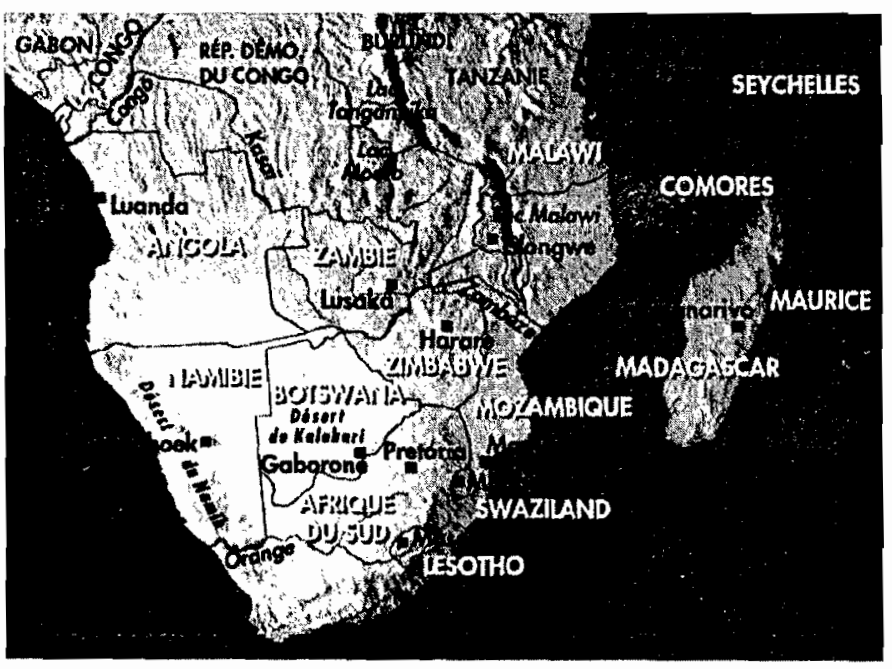




\section{Le chichewa: quelques particularités}

Le chichewa est une langue qui, d'après M. Guthrie (1948), appartient à la famille des langues bantoues. Celles-ci sont des langues qui s'étendent sur une grande partie de l'Afrique sub-saharienne, notamment au sud de l'équateur. Le chichewa fait partie du groupe Bénoué-Congo de la famille Congo-Kordofanienne et dans la classification de M. Guthrie (1948), il se situe dans la zone N 30 du groupe Nyanja. Ainsi, il a quelques caractéristiques phonétiques, prosodiques et morpho-syntaxiques qui lui sont particulières et que nous allons passer en revue.

Sur le plan prosodique, le chichewa est une langue à tons. M. Van Spaandonck (1971) définit les langues à tons comme «des langues qui présentent sur chaque syllabe un ton significativement distinctif, contrastif" (p. 31) et ce ton peut remplir une fonction lexicale, grammaticale ou syntaxique. A ce propos, le chichewa possède deux tons fondamentaux : haut $/ \%$ et bas $\%$. Selon certains linguistes, il y aurait des tons perçus comme mélodiques (c'est-à-dire ascendant $r /$ ou descendant $r /$ ) en chichewa mais ceux-ci seraient dérivés de la combinaison des deux tons fondamentaux (M. Chimombo, A. Mtenje, 1989, p. 106; S. Myers, T. Carleton, 1996, p. 68). Par ailleurs, il est à noter que dans la plupart des transcriptions, le symbole $\%$ qui signifie un ton haut est marqué alors que le symbole représentant le ton bas n'est pas marqué. Nous adoptons la même démarche dans les exemples qui suivent.

Soulignons, à titre de rappel, que les tons servent, premièrement, à distinguer les significations des mots «absolument homonymes à tous autres égards» (P. Alexandre, 1967, p. 49). Dans les paires suivantes, c'est le ton employé qui joue un rôle distinctif au niveau de la signification des mots :

\begin{tabular}{|c|c|}
\hline$k h a-l a\left[\mathrm{k}^{\mathrm{h}} \mathrm{ala}\right]=\mathrm{s}$ 'asseoir & $k^{h} \dot{a}-l a\left[\mathbf{k}^{h}\right.$ ala $]=$ braise \\
\hline$m t e-n g o[\mathrm{mt \varepsilon ngo}]=$ prix & $m t e ́-n g o[\mathrm{mtengo}]=$ arbre \\
\hline $\begin{array}{l}\text { kho-ma }\left[\mathrm{k}^{\mathrm{h}} \mathrm{oma}\right]=\text { cogner quelqu'un, } \\
\text { fixer (un clou) }\end{array}$ & $k h \dot{-}-m a\left[\mathrm{k}^{\mathrm{h}} \mathrm{Oma}\right]=\mathrm{mur}$ \\
\hline
\end{tabular}

Ces quelques exemples illustrent la pertinence des tons sur le plan lexical en chichewa, assumant les fonctions "oppositive et différenciative» selon l'appellation de M. van Spaandonck (1971, p. 37). 
Outre ce rôle, les tons semblent aussi être étroitement liés aux transformations morphosyntaxiques qui résultent du rattachement des affixes et des morphèmes divers à une racine verbale. Dans ses analyses, A. Mtenje (1987) a montré que l'ajout à la racine verbale des morphèmes de temps, de négation ou d'accord avec le complément d'objet provoque l'imposition de tons hauts sur certaines syllabes du verbe (A. Mtenje (1987) cité par M. Chimombo, A. Mtenje, 1989, p. 105). A titre d'exemple, le verbe we-re-nga [werenga], qui signifie lire en français, est d'ordinaire caractérisé par un ton bas sur toutes les syllabes. Or, le processus d'affixation des morphèmes de temps engendre l'imposition d'un ton haut sur soit la première syllabe soit la deuxième syllabe du verbe, à savoir:

Préfixe de personne morphème de temps

verbe (racine)

[1] ndi-

$n a-$

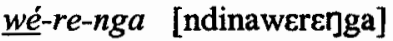

$=J$ 'ai lu.

[2] ndi-

$m a-$

we-ré-nga [ndimawerenga]

= Je lis d'habitude.

Alors qu'un ton haut s'impose sur la première syllabe du verbe après l'affixation du morphème du passé na- [na] dans l'exemple [1] ci-dessus, nous remarquons que c'est la deuxième syllabe qui acquiert un ton haut à la suite de l'agglutination du morphème d'habitude $m a$ - [ma] dans la phrase [2]. De la même manière, le rattachement d'autres morphèmes (de négation ou de futur) semble aboutir aux mêmes résultats d'imposition de tons hauts sur une syllabe du verbe:

Préfixe de personne futur verbe (racine)

[3]

\begin{tabular}{|c|c|c|}
\hline Négation & sujet & $\begin{array}{l}d z a-\quad \text { wé-re-nga } \\
=\text { Je lirai. } \\
\text { futtur verbe (racine) }\end{array}$ \\
\hline$s i-$ & $n d i-$ & $\begin{array}{l}\text { dza- wé-re-nga } \\
=\text { Je ne lirai pas. }\end{array}$ \\
\hline
\end{tabular}

[ndidzawerenga]

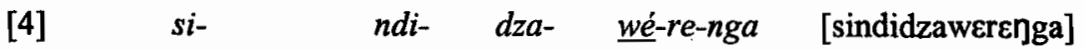
$=$ Je ne lirai pas. 
A partir des exemples cités ci-dessus, force est de conclure que les tons font partie du système lexical, morpho-syntaxique et sẻmantique du chichewa. Il nous semble donc que $P$. Alexandre (1967) a raison de déclarer que dans des langues comme celle-ci, il est impossible de communiquer sans respecter les tons, étant donné qu'il y a une prédominance de morphèmes (opérateurs grammaticaux) monosyllabiques.

Sur le plan de la phonétique, on distingue cinq sons vocaliques, à savoir : [a], [u], [i],

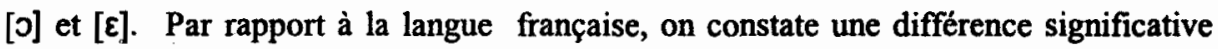
dans la mesure où celle-ci comporte seize sons vocaliques. Soit : $[i],[e],[\varepsilon],[\propto],[\mu]$, [a], [ø], [ẽ], [u], [ə], [o], [э], [a], [б] [œ] et [ã] (M. Callamand, 1981, p. 87).

Au niveau morpho-syntaxique, le chichewa est une langue dite «agglutinante». C'est-àdire qu'il s'appuie sur un système d'affixes qui fonctionne comme des indicateurs de temps verbal, d'aspect ou de personne, pour ne citer que ceux-là. Ainsi, les différentes catégories de mots (des substantifs, des adjectifs, des adverbes...) sont parfois constitués de plusieurs morphèmes ayant des fonctions syntaxiques et sémantiques variées. D'une manière générale, la configuration des morphèmes dans une structure verbale la plus complexe en chichewa peut s'analyser sur la base du modèle suivant:

\begin{tabular}{|cccccc|}
\hline $\begin{array}{c}\text { Préfixe - préfixe } \\
\text { Négation sujet }\end{array}$ aspect & temps & $\begin{array}{c}\text { complément } \\
\text { d'objet }\end{array}$ & racine & $\begin{array}{l}\text { dit d'kapplication» } \\
\text { causatif, intensif, passif }\end{array}$ \\
\hline
\end{tabular}

L'énoncé suivant, que nous empruntons à M. Chimombo et A. Mtenje (1989), sert à illustrer la configuration des morphèmes en chichewa:

Si- $\quad n d i-\quad k a-\quad n a-n g o-m u-\quad p i t-i r-\quad a$ [sindikanangomupitira]

Négation sujet conditionnel passé juste il/elle aller factitif voyelle finale = Je ne serais pas allé uniquement pour lui/elle.

D'autres exemples moins complexes que celui-ci mais qui confirmeraient quand même le caractère agglutinatif de la morphologie verbale de la langue sont les suivants: 
[5] $\mathrm{Si}-\quad t i-\quad k u-w a-d z i w-a \quad$ [sitikuwadziwa]

Négation sujet infinitif les connaître voyelle finale $=$ Nous ne les connaissons pas.

[6] Ti - kamb - iran - e [tikambiranc]

Sujet discuter réciprocité voyelle finale $=$ Que nous discutions.

Sur la base des exemples ci-dessus, nous pourrions conclure que l'agglutination est un trait inhérent à la morphologie verbale du chichewa dans la mesure où différents morphèmes se rattachent à la racine du verbe pour remplir des fonctions syntaxiques et sémantiques diverses. Aussi, nous avons vu plus haut que les transformations morphologiques du verbe engendrent souvent des modifications significatives sur le plan de réalisations tonales.

On notera qu'en chichewa, contrairement au français, les noms ne sont pas sexués. Et sur le plan syntaxique, tous les adjectifs et les autres mots qui qualifient un nom sont postposés (sauf en poésie). On aura donc :

Bambo aatali awo abvala malaya oyera ndi olemera.

[bamboataliawoavalamalajaj $\varepsilon$ randiolemera).

Traduction littérale: *Monsieur grand ce porte chemise blanche est riche. $=$ Ce grand monsieur qui porte une chemise blanche est riche.

Un trait fondamental du système morphologique du chichewa est la présence de classes nominales ainsi que des schèmes d'accord. C'est-à-dire qu'à chaque nom, singulier ou pluriel, sont associés d'une part un préfixe, et de l'autre une chaîne d'affixes d'accord (appelés aussi des préfixes de dépendance) et ces affixes sont déterminés par le préfixe nominal. G. Rebuschi (1999) définit une classe nominale comme l'ensemble des noms qui relèvent d'une classe d'accords donnée. Dans la série de noms suivante qui oppose le singulier au pluriel, on distingue un préfixe de classe (souligné) de la base nominale: 


\begin{tabular}{|c|c|}
\hline Singulier & Pluriel \\
\hline 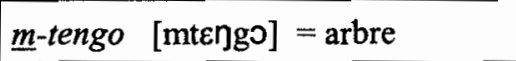 & mi-tengo [mitengo] $=$ arbres \\
\hline$\underline{m}$-phika $\left[\mathrm{mp}^{n} \mathrm{ika}\right]=$ marmite & mi-phika $\left[\mathrm{mip}^{\mathrm{h}} \mathrm{ika}\right]=$ marmites \\
\hline m-pando [mpando] = chaise & mi-pando [mipando] $=$ chaises \\
\hline$\underline{m}$-kangano [mkangano] $=$ dispute & 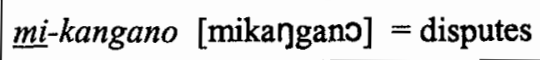 \\
\hline
\end{tabular}

Comme on peut le constater, la colonne du singulier regroupe les noms formés par le préfixe $m$ - et une racine nominale et que leur pluriel est formé par le préfixe $m i$ - [mi]. D'une manière générale, certains noms qui commencent par $m$ - au singulier constituent la classe 3 en chichewa et leurs pluriels ( $m i-$ + racine nominale) constitue la classe 4 . En outre, les classifications des noms sont déterminées par les affixes d'accord (ou schèmes d'accord) qui se rattachent aux autres éléments linguistiques dépendant du nom. Dans les exemples cités plus haut, l'affixe d'accord pour tous les noms de la classe 3 sera $u$ - [u] et que les noms de la classe 4 emploient l'affixe $i$ - [i]. Voici quelques exemples d'affixes d'accord pour les noms de ces deux classes :

\begin{tabular}{|c|c|c|}
\hline Classe & $\begin{array}{l}\text { Affixe } \\
\text { d'accord }\end{array}$ & Exemples \\
\hline \multirow[t]{2}{*}{3} & \multirow[t]{2}{*}{$\mathrm{u}-$} & $\begin{array}{l}\text { 1.Mtengo } \underline{u} \text {-wo } \underline{u} \text {-libe nthambi } \\
\text { [mtengouwoulibent }{ }^{\text {hambi] }} \\
=\text { Cet arbre n'a pas de branche. }\end{array}$ \\
\hline & & $\begin{array}{l}\text { 2. Mpando u-li apowo ndiugula } \\
\text { [mpandouliapowondiugula] } \\
=\text { La chaise qui est là, je l'achèterai. }\end{array}$ \\
\hline \multirow[t]{2}{*}{4} & \multirow[t]{2}{*}{ i- } & $\begin{array}{l}\text { 3. Mitengo } \underline{i} \text {-yo } \underline{i} \text {-libe nthambi } \\
\text { [mitengoijoilibent }{ }^{\text {nambi] }} \\
\text { = Ces arbres n'ont pas de branche }\end{array}$ \\
\hline & & $\begin{array}{l}\text { 4.Mipando } \underline{i} \text {-li apoyo ndiigula } \\
\text { [mipandoiliapojondiigula] } \\
\text { = Les chaises qui sont là, je les achèterai. }\end{array}$ \\
\hline
\end{tabular}


Il ressort de l'étude des exemples ci-dessus que la détermination d'une classe nominale pour un nom quelconque est un processus assez complexe qui exige la connaissance de la racine, des préfixes et des affixes d'accord qui correspondent au singulier ainsi qu'au pluriel. G. Rebuschi (1999) observe, à juste titre, que la connaissance de la signification du nom est déterminant puisque d'autres critères entrent en considération aussi lors de la classification (la classe 1, par exemple, comporte essentiellement des noms qui désignent des personnes ou des professions alors que la classe 12 est uniquement pour tous les noms qui comportent le diminutif $k a-[\mathrm{ka}]$ ).

En utilisant d'une part, les préfixes d'opposition singulier/pluriel et d'autre part, les affixes d'accord, on peut résumer les classes nominales du chichewa sous la forme du tableau ci-dessous :

\begin{tabular}{|c|c|c|c|c|}
\hline \multicolumn{2}{|c|}{ NO. DE CLASSE } & PREFIXE(S) DE & AFFIXE & EXEMPLE \\
\hline 1. & SING. & M-, MU-, MW- & A- & $m u-n t h u\left[\operatorname{munt}^{t^{h} u}\right]$ \\
\hline 2. & PLUR. & A- & A- & $\underline{a}-n t h u\left[\operatorname{ant} t^{h} u\right]$ \\
\hline 3. & SING. & $\mathrm{M}-, \mathrm{MU}-, \mathrm{MW}-$ & U- & $\underline{m}$-pando [mpando] \\
\hline 4. & PLUR. & MI- & I- & mi-pando [mipando] \\
\hline 5. & SING. & DZ-, KH- ... & $\mathrm{Ll}-$ & $\underline{d z \text {-ira [cira] }}$ \\
\hline 6. & PLUR. & MA- & A- & ma-zira [mazira] \\
\hline 7. & SING. & CHI- & CHI- & chi-patala [t]ipatala] \\
\hline 8. & PLUR. & ZI- & $\overline{Z I}$ & zi-patala [zipatala] \\
\hline 9. & SING. & $\mathrm{N}-, \mathrm{MPH}-, \mathrm{MB}-, \ldots$ & I- & nyumba [numba] \\
\hline 10. & PLUR. & $\overline{\mathrm{N}-}, \overline{\mathrm{MPH}-}, \mathrm{MB}-, \ldots$ & ZI- & nyumba [numba] \\
\hline \multicolumn{5}{|c|}{ 11. Cette classe semble inexistante en chichewa } \\
\hline 12. & SING. & KA- & KA- & ka-mwana [kamwana] \\
\hline 13. & PLUR. & TI- & TI- & ti-ana [tiana] \\
\hline 14. & SING. & U- & U- & $\underline{u-l a l o}[$ ulalo] \\
\hline
\end{tabular}




\begin{tabular}{|c|c|c|c|}
\hline $15 . \quad$ PLUR. & MA-(U)- & A- & ma-ulalo [maulalo] \\
\hline 16. & $\mathrm{KU}-$ (infinitif) & KU- & ku-sewera [kusewera] \\
\hline 17 & KU- & $\mathrm{KU}$ & $\underline{k u}-n y u m b a$ [kunumba] \\
\hline 18 & $\mathrm{PA} \longrightarrow$ des locatifs & PA- & pa-mpando [pampando] \\
\hline 19 & & MU- & $\begin{array}{l}\text { mu-galimoto } \\
\text { [mugalimoto] }\end{array}$ \\
\hline
\end{tabular}

Un examen du tableau fait apparaître l'organisation des classes nominales du chichewa. On dénombre 18 classes nominales étant donné que la onzième classe ne semble pas être représentée en chichewa même si celle-ci est attestée dans d'autres langues bantoues (P.J. Kishindo, 1999, p. 66).

Aussi, force est de souligner que même si la classification semble reposer sur l'opposition singulier/pluriel, ce critère ne joue aucun rôle quand il s'agit de noms qui n'ont pas de singulier (par exemple les noms de liquides) ou ceux qui n'ont pas de pluriel (les noms abstraits). Egalement, la classe des infinitifs (des verbes à la forme infinitive $k u-[\mathrm{ku}])$ n'aurait pas de pluriel.

Quant au sytème verbal du chichewa, il importe de faire remarquer, premièrement, qu'il n'y a pas de conjugaisons, étant donné que la racine verbale reste invariable. $C^{\prime}$ 'est uniquement le sujet personnel qui varie. Ainsi, en utilisant le verbe $k u$-dya [kudja] qui signifie manger en français, on aura (au présent):

$\begin{array}{lc}\text { Ndi- } k u-d v a \text { [ndikudja] } & =\text { Je mange } \\ U-\underline{k u-d v a} \text { [ukudja] } & =\text { Tu manges } \\ \text { A- } \underline{k u-d y a} \text { [akudja] } & =\text { Il/Elle mange } \\ & \text { Ils /Elles mangent } \\ T i-\underline{k u-d v a} \text { [tikudja] } & =\text { Nous mangeons } \\ M u-\underline{k u-d v a} \text { [mukudja] } & =\text { Vous mangez }\end{array}$

Même si l'on effectue un changement de temps verbal des énoncés ci-dessus, on aboutira au même résultat. Soit:

$$
\text { Sujet + morphème de temps + racine verbale }
$$

Deuxièmement, on distingue trois temps verbaux en chichewa, à savoir: le présent, le passé et le futur. On associe un affixe à chacun de ces temps verbaux qui se rattache à 
la racine verbale. Les trois affixes représentant les trois temps verbaux sont les suivants :

- Présent : $\quad-k u-[\mathrm{ku}]$

- Passé -na-[na]

- Futur -dza-[cta]

Exemple : $k u-d y a$ [kudja] (manger) :

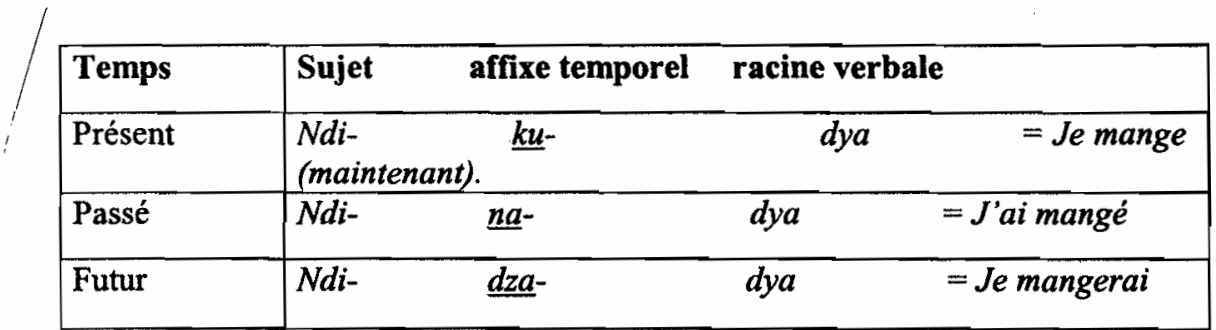

A ces trois formes s'ajoutent des adverbes temporels et des locutions qui apportent des précisions à la chronologie des faits et des événements, par exemple:

- $\quad$ lero [lero]: aujourd'hui;

- mawa [mawa]: demain;

- sabata ya mawa [sabatajamawa]: la semaine prochaine

ainsi que des morphèmes pour exprimer les formes aspectuelles. A ce sujet, $F$. Chilipaine (1994) distingue les formes aspectuelles suivantes en chichewa:

- $\quad-l i$ [li]: l'inaccompli au présent. Exemple: Ndili kulima [ndilikulima] = Je suis en train de cultiver.

$-n a+l i$ [nali]: l'inaccompli au passé. Exemple : Ndinali kulima [ndinalikulima] =Je cultivais/J'étais en train de cultiver.

- $\quad-a$ - [a] : l'accompli. Exemple : Ndalima munda [ndalimamunda] $=J$ 'ai cultivé un champ.

- $\quad-z i+d z a$ [zidza]: l'habitude au futur (ce morphème est souvent accompagné d'un adverbe, par exemple kawirikawiri [kawirikawiri] - souvent).

Exemple: Ndizidzasewera kawirikawiri [ndizidzasewErakawirikawiri]

= Je jouerai souvent. 
Pour exprimer l'antériorité en chichewa, on s'appuie sur le morphème -ta- [ta]. Exemple:

Atalemba kalata, anaitumiza [atalembakalata anajitumiza].

= Après avoir écrit une lettre, il l'envoya.

Dans cet énoncé, le morphème -ta- dans atalemba [atalemba] exprime l'antériorité de l'action d'écrire par rapport à celle d'envoyer.

Cette description du système des temps verbaux du chichewa donne une idée de son organisation même si nous sommes conscient du fait que cette présentation est schématique. Tout de même, on peut conclure qu'en chichewa, on distingue trois temps verbaux (le présent, le futur et le passé) ainsi que des morphèmes aspectuels, exprimant les aspects comme l'antériorité, l'habitude, l'accompli, pour ne citer que quelques exemples.

\section{Remarques conclusives}

On ne peut manquer de constater que nous préconisons une démarche contrastive dans l'enseignement langagier. Or il importe de signaler que nous sommes conscient des objectifs mais aussi des limites de la linguistique contrastive. L'ambition de l'analyse contrastive au départ était la comparaison de deux langues, et surtout de leurs diffẻrences structurales, afin de permettre l'élaboration des méthodes d'enseignement mieux adaptées aux difficultés spécifiques des apprenants d'une langue maternelle donnée (F. Debyser, 1970, p. 31). Il s'agissait de décrire dans l'objectif soit de prévenir soit de corriger les fautes imputables à l'influence de la langue maternelle. Toutefois, comme le constatent $H$. Besse et $R$. Porquier (1991, p. 204), divers constats empiriques ont contribué à remettre en question les présupposés théoriques de l'analyse contrastive a priori ainsi que sa pertinence pour l'enseignement:

- beaucoup d'erreurs prévues par une analyse contrastive ne se produisent pas, ou rarement, ou seulement à certains stades d'apprentissage, ou de façon aléatoire selon les individus, les circonstances, les méthodes ou les exercices. D'autres erreurs ne se produisent qu'à un niveau avancé;

- il y a des erreurs qu'on peut considérer comme «universelles», c'est-à-dire qu'elles sont commises par des apprenants de langues maternelles différentes;

- quelques unes des erreurs attribuées à l'interférence se rencontrent aussi chez des enfants natifs soit dans certaines variètés ou normes de la langue cible. 
Cela étant dit, on reconnaît que ces critiques n'invalident pas le principe de l'analyse contrastive de deux langues dans la mesure où une étude contrastive a posteriori a le mérite de traiter des transferts effectivement observés chez les apprenants et cette approche paraît plus opératoire que celle de l'analyse a priori puisque celle-là "entre dans le cadre empirique de l'analyse des erreurs" (H. Besse, R. Porquier, 1991, p. 206).

De son côté, F. Debyser (1970) met l'accent sur la valeur prédictive de l'analyse contrastive. Il précise qu'une comparaison de deux langues «aura une certaine valeur prédictive puisqu'elle mettra en évidence les aires interférentielles. Ainsi, chaque fois qu'il y aura une structuration plus complexe en $L 2$ qu'en $L 1$, on peut être à peu près sûr qu'il y aura des interférences, quel que soit le niveau d'organisation du langage considéré (phonologique, morphosyntaxique ou lexical)» (p. 54). C'est dans ce sens que nous évoquerons des comparaisons entre certains aspects du chichewa et du français à des fins didactiques. En accord avec J-P. Cuq (1996), nous pensons que si cette approche n'aide pas à déterminer à coup sûr pourquoi un apprenant commet telle ou telle faute, elle permet au moins «de systématiser a posteriori, par la comparaison de micro-structures, des constatations empiriques qui ont des probabilités importantes de renouvellement chez des sujets comparables» (p. 44). Dans notre contexte, il nous semble que parmi les micro-structures susceptibles de poser des problèmes chez les apprenants chichewaphones, on trouvera :

- les conjugaisons des verbes en français,

- l'emploi des temps verbaux,

- la notion de genre des substantifs,

- l'accord de genre et de nombre entre le nom ou le pronom et le verbe, l'adjectif et le participe.

Il est évident que ces aspects de la langue française exigeront des efforts cognitifs considérables chez les apprenants chichewaphones dans la mesure où leur apprentissage s'apparentera à "une activité de résolution de problème» (G. Vigner, 1990, p. 39). L'enjeu dans la didactique du français sera donc la mise en cuvre d'un enseignement qui prend en considération ces écarts entre les deux langues. 


\section{Bibliographie}

Alexandre, P. 1967. Langues et langage en Afrique noire. Paris: Payot.

Besse, H., Porquier R. 1991. Grammaire et didactiques des langues langues. Paris: Hatier/Didier/CREDIF.

Bresnan, J., Mchombo S.A. 1987. Topic, pronoun and agreement in Chichewa, Language, 63. 4, 741-782.

Callamand, M. 1981. Méthodologie de l'enseignement de la prononciation. Paris: CLE International.

Chilipaine, F. 1994. Morpho-syntaxique du verbe: français-chichewa (Malawi). Paris: Editions L'Harmattan.

Chimombo, M., Mtenje A. 1989. Interaction of tone, syntax and semantics in the acquisition of chichewa negation. Studies in African Linguistics, 20, 2, 103-150.

Cuq J-P. 1996. Une introduction à la didactique de la grammaire en français langue étrangère. Paris.

Debyser, F. 1970. La linguistique contrastive et les interférences, Langue française. pp. 31-61.

Guthrie, M. 1948. The classification of Bantu languages. London: Dawsons of Pall Mall.

Kishindo, P.J. 1999. Ikyangonde: a preliminary analysis, Journal of Humanities. 13, pp. 59-86.

Myers, S., Carleton T. 1996. Tonal transfer in Chichewa. Phonology, 13, 1, 39-72.

Rebuschi, G. 1999. Classes de langues et genres dans les langues bantoues, Faits de langue, pp. 185-196.

Roulet, E. 1995. Peut-on intégrer l'enseignement-apprentissage décalé de plusieurs, Etudes de Linguistique Appliquée, 98, 113-118.

Van Spaandonck, M. 1971. L'analyse morphotonologique dans les langues bantoues.

Paris: SELAF.

Vigner, G. 1990. Activité d'exercice et acquisitions langagières, Acquisition et utilisation d'une langue étrangère. L'approche cognitive. Hachette, pp. 134-146.

Widdowson, H.G. 1981. Une approche communicative de l'enseignement. Paris: Hachette/CREDIF.

Department of French

Chancellor College

University of Malawi

P.O. Box 280, Zomba

Malawi

alipenga@chanco.unima.mw 